\title{
Celebrating graphene
}

\section{Graphene - a form of carbon isolated only six years ago - is the topic of this year's Nobel Prize for Physics, giving hope for future applications of this intriguing material in the field of photonics.}

Given the explosion of research into graphene over the past few years, it seems fitting that this year's Nobel Prize for Physics has been awarded to the two scientists who first isolated and explored this intriguing material ${ }^{1}$. One only has to look at the growing number of research papers concerned with graphene and the number of citations that they are receiving to see just how influential this discovery has been.

The successful fabrication and characterization of graphene - a singleatom-thick sheet of carbon atoms arranged in a hexagonal lattice - was first reported by Andre Geim and Konstantin Novoselov and their co-workers from the University of Manchester, UK, in $2004^{2}$. This paper has so far received over 3,000 citations, according to the ISI web of knowledge.

The publication of this research started a race to experimentally explore this new form of carbon and its intriguing electronic, mechanical and optical properties. For example, the following year the unusual quantum Hall effect in graphene was demonstrated independently by groups at Manchester and Colombia University and co-published in the same issue of Nature in $2005^{3,4}$. Today, graphene's high electrical conductivity (a resistance of $31 \Omega \mathrm{m}^{-2}$ ), broadband transparency (a flat response across the visible range, with each layer absorbing only $\sim 2.3 \%$ of the incident light), high flexibility and great strength (exceeding that of steel) are now proving enticing for a range of potential applications, of which many are strongly related to photonics. Indeed, one of the most promising applications of graphene is as a thin, robust and highly transparent electrode for a variety of optoelectronic devices ranging from solar cells to displays and light emitters.

In principle graphene could also be an important alternative to indium tin oxide, which, despite being expensive and brittle, is today's material of choice for transparent electrodes. Given the current level of interest in developing flexible organic/polymer-based light emitters, displays and photovoltaics, the emergence of graphene as a potentially more robust and cost-effective alternative to indium tin oxide could turn out to be an important and timely development. The roll-to-roll production of graphene sheet electrodes for surface areas as large as 30 square inches was

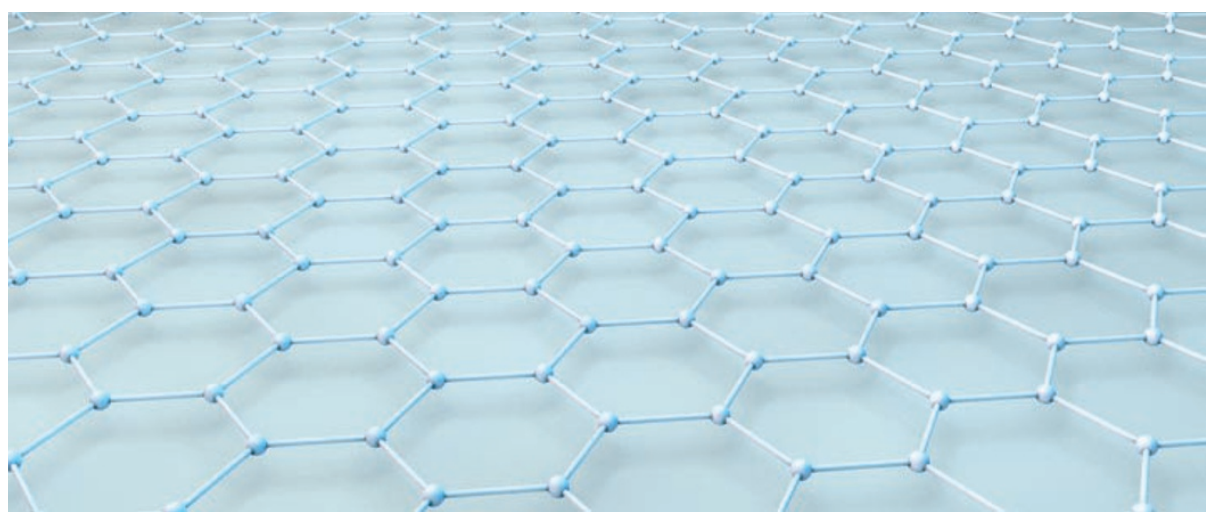

recently achieved ${ }^{5}$, suggesting that it may not be long before graphene is considered as a practical electrode technology for use in commercial devices.

Interest in graphene from the photonics community does not stop here, as evidenced by our recent review on graphene photonics and optoelectronics, published only two months ago ${ }^{6}$. Researchers have already shown that graphene can successfully be used as a saturable absorber for realizing ultrafast mode-locked fibre lasers emitting picosecond and femtosecond duration pulses at repetition rates of several megahertz. Graphene is also being considered for use in optical limiters, photodetectors and optical frequency converters. It is likely that this Nobel Prize will inspire more optical scientists to further investigate this intriguing material, giving hope for its use in a wealth of future photonic applications.

Interestingly, it is not the first time that scientists working in the UK have received a Nobel Prize for their research on carbon allotropes (carbon atoms arranged in different structural forms). In 1996, Harry Kroto from the University of Sussex, together with Robert Curl and Richard Smalley from Rice University in the USA, shared the Nobel Prize for Chemistry for their discovery of buckminsterfullerene - a ball-shaped cluster of 60 carbon atoms. However, in this case there was a gap of 11 years between their initial discovery (published in Nature in $1985^{7}$ ) and the actual Nobel Prize.

Indeed, the timescale of just six years between the initial work of Geim and Novoselov and their award is astonishingly short, particularly when compared with other Nobel Prizes. Historically, the Nobel Committee will often not award a prize until the impact a scientific breakthrough has on society is clearly visible and wellestablished. For example, the researchers who performed the initial research on magnetic resonance imaging in the 1970s had to wait over twenty years before they received the Nobel Prize for Medicine and Physiology in 2003. And last year's Nobel Prize for Physics, awarded to Charles Kao for the realization of low-loss silica optical fibre, and to Willard Boyle and George Smith for the CCD image sensor, concerns research that all took place in the 1960s. The contrast between the 2009 and the 2010 awards is striking. As we said when the 2009 Nobel Prize for Physics was presented $^{8}$, in the 40 years since the initial fibre and CCD research was performed, both technologies have matured to achieve widespread adoption, underpinning modern telecommunications and digital imaging. In contrast, the situation for this year's Nobel Prize for Physics is rather different, as it celebrates a young, exciting area of materials research that today is still far from maturity.

\footnotetext{
References

1. http://nobelprize.org/nobel_prizes/physics/laureates/2010/

2. Novoselov, K. S. et al. Science 306, 666-669 (2004)

3. Novoselov, K. S. et al. Nature 438, 197-200 (2005).

4. Zhang, Y., Tan, Y.-W., Stormer, H. L. \& Kim, P. Nature 438, 201-204 (2005).

5. Bae, S. et al. Nature Nanotech. 5, 574-578 (2010).

6. Bonaccorso, F., Sun, Z., Hasan, T. \& Ferrari, A. C. Nature Photon. 4, 611-622 (2010)

7. Kroto, H. W., Heath, J. R., O’Brien, S. C. O., Curl, R. F. \& Smalley, R. E. Nature 318, 162-163 (1985)

8. Nature Photon. 3, 605 (2009).
} 